The most intense ring of $\mathrm{MgO}$ is found, though faint, in the pattern of the specimen left in the air at room temperature; likewise, the most intense ring of $\mathrm{Mg}$ is soen faintly in the pattern of the specimen heated at $400^{\circ} \mathrm{C}$. It has therefore been concluded that magnesium metal is masked with a thin film of magnesium oxide ( $\mathrm{MgO}$ ) even at room temperature, and the film is still quite thin even after heating at $400^{\circ} \mathrm{C}$. Such a thin film, $10 \mathrm{~A}$. or so in thickness, protects the metal and keeps it lustrous for a long time.

Although it is very difficult to obtain the patterns of the aluminium oxides by the reflection method'. we succeeded in producing them by making deep ditches on the metal. Precisely as in the case of magnesium, it was possible to verify that the surface of aluminium at room temperature is covered with a film of $\gamma \cdot \mathrm{Al}_{2} \mathrm{O}_{3}$, while the specimen heated at $500^{\circ} \mathrm{C}$. is covered with a film of $\alpha-\mathrm{Al}_{2} \mathrm{O}_{3}$.

With beryllium left exposed at room temperature, the pattern obtained from the surface precludes the possibility of even a trace of rings belonging to the oxide BeO.

Full details of these experiments will bo published in the Scientific Papers of our Institute. IcHirô IrTAKa.

Instituto of Physical and Shigeto Yamagucir.

Chemical Research, Toliyo.

${ }^{1}$ Preston, G. D., and Bircumshaw, L. L., Phil. Mag., 22, 654 (1936).

\section{Decomposition of Hydrogen Peroxide by Catalase}

THAT the catalytic decomposition of hydrogen peroxide by catalase requires the presenco of molecular oxygen was demonstrated by a number of manometric experiments previously described ${ }^{1}$. Being, however, well aware of certain difficulties in this kind of work, we have given a detailed account of our methods so that our experiments could easily be repeated.

The failure by Weiss and Weil-Malherbe ${ }^{2}$ to confirm our results can only be ascribed to some defects in their experimental procedure and to the fact that they have not closely followed the recommendations put forward in our paper.

Our criticism ${ }^{3}$ of experiments with luminescent bacteria was not solely due to the purely qualitative character of this work-4, but mainly to the fact that under the conditions of these experiments even a 90 per cent inhibition of catalase could not be detected.

That the curves of inhibition shown in our paper are not of autocatalytic typo can be explained by the instability of pure catalase at high dilutions $(0.0005 \mathrm{mgm}$. per flask) used in our experiments.

Moreover, our manometric experiments, which we have repeated several times since the publication of our paper, are in complete agreement with the results wo have obtained from the study of catalase inhibited by sodium azide or by hydroxylamine ${ }^{5}$. Both these inhibitors combine reversibly with catalase giving well-defined compounds.

It is, however, important to remember that freo catalase and azide- or hydroxylamine-catalase are very similar compounds displaying the same general properties. Thus, their iron is in the trivalent state, they show a typical methrmoglobin-like absorption spectrum, they share the remarkable property of not. being reduced by a powerful reducer like sodium hyposulphite and they react with hydrogen peroxide.

While the reaction of free catalase with hydrogen peroxide is extremely rapid and therefore inaccessiblo to spectroscopic study, that of azide- or hydroxyl. amine-catalase is on the contrary slow and can easily be followed spectroscopically. The study of this reaction clearly shows that azide-catalase treated with hydrogen peroxide undergoes reduction which is marked by a distinct change in its colour and its absorption spectrum and by the appearance of the property, shared with other divalent haematin compounds, of combining with carbon monoxide. On the other hand, the reduced azide-catalase cannot be oxidized by hydrogen peroxide although it can be oxidized by molecular oxygen, which in this reaction is reduced not to hydrogen peroxide but to water. In this. respect catalase, unlike many autoxidizable compounds, resembles cytochrome oxidase and probably polyphenol oxidase which on reoxidation reduce oxygen to water. Azide and hydroxylamine inhibit. the activity of catalase only because they slow down the reoxidation of ferrous catalase by oxygen.

Our conclusion that catalatic decomposition of hydrogen peroxide is brought about by a constant reduction of catalaso iron by hydrogen peroxide and its reoxidation by molecular oxygen is thereforo well supported by two independent experimental ap. proaches to the problem, namely the manometric and spectroscopic studies of the enzyme. The importance of specific inhibitors and of spectroscopic methods in the study of enzymes is now universally recognized and can scarcely bo over-estimated. In very few cases, however, can the importance of these methods be so clearly demonstrated as in the study of catalase.

Considering that a pure or even crystalline catalase showing strong colour and absorption spectrum can be so easily prepared, the workers ${ }^{2}$ interested in the intimate mechanism of catalatic activity should attempt to prepare such an enzyme and to acquiro thus a first-hand knowledge of its properties.

The fact that the results of our experiments do not agree with certain theoretical considerations:" concerning the possible mechanism of catalytic activity of this enzymo suggests only that these considerations require some revision.

Molteno Institute,

D. KEILN.

University,

Cambridge.

Dec. 13.

1 Keilin, D., and Hartree, E. F., Proc. Roy. Soc., B, 124, 397 (1938). 'Weiss, J., and Weil-Malherbe, H., NarcRe, 144, 866 (1939).

- Keilin, D., and Hartree, E. F., NATCRE, 144, 787 (1939).

4 Johnson, F. H., and van Schouvenburg, N $\triangle$ TERE, 144, 634 (1939).

s Keilin, D., and Hartree, E. F., Proc. Roy. Soc., B, 121, 173 (1936).

- Weiss, J., J. Phys. Chem., 41, 1107 (1937).

\section{Evocation in the Chick}

Work on the evocation of neural tissue in vertebrate embryos by chemically prepared substances has hitherto been confined to the Amphibia ${ }^{2}$. But since in the induction of neural tissue in general the chick has shown considerable similarities to the newt, especially in the inducing activity of its organizer when coagulated ${ }^{2}$, and in the inducing activity of fully differentiated tissues ${ }^{3}$, there was reason to 